\title{
Avaliação do consumo alimentar de pacientes com doença renal crônica em hemodiálise
}

\author{
Assessment of dietary intake of patients with chronic kidney disease on hemodialysis
}

\author{
Alisson Diego Machado ${ }^{1}$, Ana Paula Bazanelli², Rosana Farah Simony ${ }^{3}$
}

\begin{abstract}
RESUMO
Objetivo: Avaliar o consumo de energia, proteína, fósforo e potássio de pacientes com doença renal crônica em hemodiálise.

Materiais e Métodos: Foi realizado um estudo transversal com 34 pacientes em hemodiálise. Foi aplicado, por meio de entrevista, um questionário para a identificação das características clínico-demográficas dos pacientes. $O$ método utilizado para a avaliação do consumo alimentar foi o registro alimentar de 3 dias. Os níveis séricos de fósforo e potássio e o valor do Kt/ $\mathrm{V}$ foram obtidos por meio de consulta ao prontuário dos pacientes.

Resultados: O consumo médio de energia, proteína, fósforo e potássio correspondeu a 19,0 kcal/kg, 0,9 g/kg, 612,5 mg e 1400,2 mg, respectivamente, sendo inferior às recomendações estabelecidas. A diferença na ingestão foi observada apenas pela variável sexo $(p<0,05)$. Não houve correlação estatisticamente significativa entre a inadequação da diálise e o baixo consumo de nutrientes e entre o consumo de fósforo e potássio e seus níveis séricos.

Conclusão: $O$ consumo alimentar dos pacientes apresentou-se inadequado, ressaltando a necessidade de realização de medidas de educação nutricional para que a ingestão dos mesmos se adeque às recomendações e as comorbidades decorrentes do consumo inadequado sejam evitadas.
\end{abstract}

Palavras-chave: consumo de alimentos; insuficiência renal crônica; diálise renal.

\begin{abstract}
Objective: To assess the dietary intake of energy, protein, phosphorus and potassium of patients with chronic kidney disease on hemodialysis.

Materials and Methods: It was performed a cross-sectional study with 34 patients on hemodialysis. A questionnaire was applied by interview to identify the clinical and demographic characteristics of patients. The method used for the assessment of dietary intake was the 3-day food record. The serum levels of phosphorus and potassium and the value of $K t / N$ were obtained by consultation on patients' charts.

Results: The average intake of energy, protein, phosphorus and potassium corresponded to $19.0 \mathrm{kcal} / \mathrm{kg}, 0.9 \mathrm{~g} / \mathrm{kg}$, $612.5 \mathrm{mg}$ and $1400.2 \mathrm{mg}$, respectively, which is lower than the established recommendations. The difference on intake was observed only for the gender $(p<0.05)$. There was no statistically significant correlation between inadequate dialysis and low intake of nutrients and between the intake of phosphorus and potassium and its serum levels.

Conclusions: The dietary intake of patients have presented as inadequate, emphasizing the need to carry out nutritional actions, so that the intake of the patients can suit the recommendations and the comorbidities resulting from the inadequate intake can be reduced.
\end{abstract}

Keywords: food consumption; chronic renal insufficiency; renal dialysis.

\footnotetext{
${ }^{1}$ Acadêmico do Curso de Nutrição da Universidade Presbiteriana Mackenzie.

${ }^{2}$ Nutricionista. Doutora em Nutrição pela Universidade Federal de São Paulo. Docente dos Cursos de Nutrição e Tecnologia em Gastronomia da Universidade Presbiteriana Mackenzie e do Curso de Nutrição das Faculdades Metropolitanas Unidas.

${ }^{3}$ Nutricionista. Doutora em Medicina (Endocrinologia Clínica) pela Universidade Federal de São Paulo. Docente do Curso de Nutrição da Universidade Presbiteriana Mackenzie;
} 


\section{INTRODUÇÃO}

A doença renal crônica (DRC) é considerada um problema emergente de saúde pública mundial, devido ao rápido aumento da sua prevalência nos últimos anos, com o agravante de que, na maioria das vezes, a mesma só é diagnosticada nos seus estágios mais graves $^{1}$. De acordo com o Censo Brasileiro de Diálise Crônica de 2012, o número estimado de pacientes em terapia renal substitutiva no Brasil corresponde a 97.586. O número de óbitos decorrentes da doença foi de 18.333 , o que equivale a $18,8 \%$ de mortalidade bruta $^{2}$.

Segundo a diretriz da Kidney Disease Outcomes Quality Initiative, podem ser considerados portadores de DRC adultos que apresentem taxa de filtração glomerular menor que $60 \mathrm{~mL} / \mathrm{min} / 1,73 \mathrm{~m}^{2}$, ou com taxa superior à preconizada, desde que associada a um marcador de lesão renal há pelo menos três meses ${ }^{3}$.

A DRC prejudica o funcionamento adequado de outros órgãos, uma vez que os rins são fundamentais para a homeostase corporal. Dentre as principais complicações que acometem os portadores dessa doença estão a desnutrição energético-proteica (DEP) e a alteração no metabolismo de minerais ${ }^{4}$.

A DEP é um dos distúrbios nutricionais mais prevalentes em pacientes em hemodiálise, estando relacionada com o aumento das taxas de morbidade e mortalidade. Sua etiologia é multifatorial e inclui aspectos relacionados tanto ao consumo alimentar insuficiente em energia e nutrientes quanto às alterações hormonais e metabólicas que podem ocasionar um aumento do catabolismo energético e/ou proteico, como perda de nutrientes pelo dialisato, bioincompatibilidade das membranas dos filtros de diálise, resistência à ação da insulina e do hormônio do crescimento, acidose metabólica, presença de comorbidades e inflamação ${ }^{5}$. Para tanto, é recomendada a ingestão de $35 \mathrm{kcal} / \mathrm{kg} / \mathrm{dia}$ para pacientes com até 59 anos de idade e de 30 a 35 $\mathrm{kcal} / \mathrm{kg} /$ dia para aqueles com idade igual ou superior a 60 anos. Para proteína, recomenda-se a ingestão de 1,2 $\mathrm{g} / \mathrm{kg} / \mathrm{dia}^{6}$.

Os minerais que devem ter seus níveis séricos controlados rotineiramente em portadores de DRC são o fósforo e o potássio. A hiperfosfatemia está entre os fatores que contribuem para o desenvolvimento de hiperparatireoidismo secundário nesses pacientes ${ }^{7}$, que por sua vez favorece o aumento do gasto energético de repouso dos mesmos, contribuindo para o desenvolvimento de desnutrição ${ }^{8}$. Em função dos níveis séricos elevados de fósforo frequentemente faz-se necessária a utilização de quelantes desse mineral para a redução de sua absorção intestinal ${ }^{9}$. A hipercalemia está associada ao risco de morte aumentado em portadores de DRC, uma vez que em níveis circulantes elevados pode levar à arritmia cardíaca ${ }^{10}$. Os níveis séricos de fósforo devem ser de 3,5 a 5,5 mg/dL, sendo que seu consumo diário deve estar entre 800 e 1000 $\mathrm{mg}^{6}$. A calemia deve estar entre 3,5 e $5,0 \mathrm{mg} / \mathrm{dL}^{6}$, sendo que a ingestão de potássio deve ser de 50 a 70 $\mathrm{mEq} / \mathrm{dia}$, o que equivale a 1950 a $2730 \mathrm{mg}^{11}$.

Considerando a importância do acompanhamento nutricional para a terapia e promoção de melhor qualidade de vida desses pacientes, o presente estudo teve por objetivo avaliar o consumo de energia, proteína, fósforo e potássio de portadores de DRC em hemodiálise.

\section{MATERIAIS E MÉTODOS}

Foi realizado um estudo transversal com 34 pacientes em hemodiálise - sessões de quatro horas, realizadas três vezes por semana - atendidos em uma clínica de nefrologia localizada no município de Guarulhos, São Paulo, durante os meses de janeiro e fevereiro de 2013. Foram inclusos pacientes com idade igual ou superior a 18 anos e que estavam em tratamento há pelo menos seis meses.

Por meio de entrevista, durante a sessão de hemodiálise, foi aplicado um questionário para a identificação das características clínico-demográficas dos pacientes, com perguntas referentes à idade, sexo, peso seco, estatura, escolaridade, estado civil, raça, renda mensal familiar, período e tempo de tratamento e tipo de pagamento. Os níveis séricos de fósforo e de potássio foram obtidos por meio de consulta ao prontuário dos pacientes, sendo que esses exames são realizados no início de cada mês. No prontuário também foi coletado o valor da adequação da diálise $(\mathrm{Kt} / \mathrm{V})$ do 
período em que o estudo foi realizado.

Após a entrevista cada participante recebeu três registros alimentares e foram orientados individualmente

a preencher os mesmos com todos os alimentos/preparações e bebidas consumidos ao longo do dia, em três momentos da semana, sendo um dia em que houve e em um dia em que não houve a sessão de hemodiálise, ambos durante a semana (segunda à sexta), e em um domingo, por ser um dia atípico. Foi estabelecido o prazo de duas semanas para a devolução dos mesmos.

Os registros alimentares foram entregues a 145 pacientes. Contudo, apenas 43 os devolveram, sendo que destes, 9 pacientes foram excluídos do estudo devido à inconsistências no preenchimento dos registros.

Para a classificação do estado nutricional calculou-se o índice de massa corporal (IMC). Os pacientes com idade inferior a 60 anos foram classificados de acordo com o preconizado pela World Health Organization ${ }^{12}$, sendo que IMC $<16,0 \mathrm{~kg} / \mathrm{m}^{2}$ representa magreza grau III; $16,0-16,9 \mathrm{~kg} / \mathrm{m}^{2}$, magreza grau II; $17,0-18,4 \mathrm{~kg} / \mathrm{m}^{2}$, magreza grau I; $18,5-24,9 \mathrm{~kg} / \mathrm{m}^{2}$, eutrofia; 25,0-29,9 $\mathrm{kg} / \mathrm{m}^{2}$, sobrepeso; $30,0-34,5 \mathrm{~kg} / \mathrm{m}^{2}$, obesidade grau I; $35,0-39,9 \mathrm{~kg} / \mathrm{m}^{2}$; e $\geq 40,0 \mathrm{~kg} / \mathrm{m}^{2}$, obesidade grau III. Os pacientes com idade igual ou superior a 60 anos foram classificados segundo o proposto pela Organização Pan-Americana da Saúde ${ }^{13}$, sendo que $I M C<23,0 \mathrm{~kg} / \mathrm{m}^{2}$ indica baixo peso; 23,0-27,9 kg/m², peso normal; 28,0$29,9 \mathrm{~kg} / \mathrm{m}^{2}$, sobrepeso; e $>30,0 \mathrm{~kg} / \mathrm{m}^{2}$, obesidade. Devido às diferenças nos pontos de corte estabelecidos, no presente estudo os pacientes que apresentaram magreza/baixo peso foram inclusos na categoria baixo peso; os que estavam na faixa de eutrofia/peso normal na categoria peso adequado; e aqueles que apresentaram sobrepeso ou obesidade na categoria excesso de peso.

Foi calculado o peso ajustado dos pacientes que, de acordo com o IMC, apresentaram percentual de adequação do peso inferior a 95\% ou superior a $115 \%$. Primeiramente foi calculado o peso ideal dos mesmos, de acordo com a fórmula: peso ideal $(\mathrm{kg})=[\mathrm{IMC}$ desejado $x$ estatura $\left.(m)^{2}\right]$.

O IMC desejado utilizado no estudo foi o IMC médio da classificação imediatamente anterior ao atual, no caso dos pacientes com sobrepeso ou obesidade; e o IMC médio imediatamente superior para os pacientes com baixo peso.

A adequação do peso atual em relação ao ideal foi calculada a partir da seguinte equação: adequação do peso $(\%)=($ peso atual $x$ 100 $) /$ peso ideal. $O$ peso ajustado foi obtido por meio da equação proposta pela National Kidney Foundation ${ }^{6}$ : peso ajustado $(\mathrm{kg})=[($ peso ideal - peso atual) x 0,25] + peso atual.

Os alimentos/preparações e bebidas consumidos foram convertidos em gramas, utilizando-se a Tabela para Avaliação de Consumo Alimentar em Medidas Caseiras $^{14}$. O consumo foi calculado pela utilização do software Avanutri Online ${ }^{\circledR}$ e comparado com as recomendações de energia, proteína e fósforo preconizadas pela National Kidney Foundation ${ }^{6}$ e de potássio, segundo Fouque et al. $^{11}$. O consumo foi apresentado em média e desvio-padrão $(m \pm d p)$, de acordo com as variáveis idade, sexo, escolaridade, estado civil, renda, estado nutricional, período e tempo de tratamento e pagamento. Para fins de análise estatística, as variáveis idade, escolaridade, estado civil e raça foram agrupadas em categorias dicotômicas.

Para a identificação dos alimentos que mais contribuíram para o consumo de fósforo e potássio utilizou-se a seguinte equação, de acordo com o método proposto por Block et al. ${ }^{15}$ : \% de contribuição do nutriente a pelo alimento $\mathrm{z}=$ (quantidade do nutriente a fornecida pelo alimento $z \times 100$ )/quantidade do nutriente a fornecida por todos os alimentos.

Foi elaborada uma lista de contribuição percentual dos alimentos, cuja somatória totalizou ao menos $95 \%$ para cada um dos micronutrientes avaliados.

Foi aplicado o teste de normalidade de KolmogorovSmirnov para as variáveis em estudo. Uma vez que a distribuição dos valores de consumo foi normal, a diferença de acordo com as variáveis em estudo foi calculada pelo teste t de Student para as variáveis com categorias dicotômicas e pela análise de variância (ANOVA) para as variáveis com três categorias, sendo significativos os valores de $p<0,05$. A diferença entre variáveis qualitativas foi calculada pelo teste exato de Fisher. O coeficiente de correlação entre o consumo de 
fósforo e potássio e seus respectivos níveis séricos, bem como entre o consumo de energia, proteína, fósforo e potássio e o Kt/V , foi obtido pela correlação de Pearson. As análises estatísticas foram calculadas pela utilização do software SPSS, versão 21.0.

O estudo foi aprovado sob número CAAE 02780712.6.0000.0084. Foram seguidas as determinações da Resolução n 196/96, do Conselho Nacional de Saúde, sendo que a coleta de dados foi realizada após a assinatura do Termo de Consentimento Livre e Esclarecido por todos os participantes do estudo.

\section{RESULTADOS}

Foram avaliados 34 pacientes, com idade média de $59,4 \pm 9,9$ anos, sendo $64,7 \%$ dos participantes do sexo masculino e $35,3 \%$ do sexo feminino.

A maioria dos pacientes possuíam até 8 anos de estudo, eram casados, de raça branca, com renda mensal familiar de 1 a 3 salários-mínimos, apresentando peso adequado, com realização da diálise no período noturno, com tempo de tratamento de 6 a 18 meses e que utilizam o Sistema Único de Saúde (SUS) como forma de pagamento (tabela 1).

Em relação ao estado nutricional, verificou-se que as prevalências de baixo peso e de peso adequado corresponderam a $5,6 \%$ e $44,4 \%$ para os pacientes adultos e $31,3 \%$ e $56,2 \%$ para os idosos, respectivamente, contudo sem diferença estatisticamente significativa $(p=0,078$ e $p=0,732$, respectivamente). O percentual de adultos com excesso de peso $(50,0 \%)$ foi estatisticamente diferente $(p=0,030)$ em relação aos pacientes idosos (12,5\%).

O consumo médio diário de energia correspondeu a

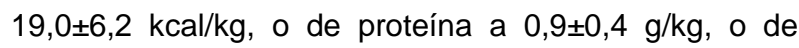
fósforo a $612,5 \pm 212,6 \mathrm{mg}$ e 0 de potássio a $1400,2 \pm 455,3 \mathrm{mg}$, sendo esses valores considerados inferiores às recomendações estabelecidas para portadores de DRC em hemodiálise.

A diferença entre a ingestão alimentar foi observada apenas para a variável sexo, na ingestão de proteína, fósforo e potássio, sendo que os pacientes do sexo masculino apresentaram ingestão estatisticamente superior em relação a esses nutrientes (tabela 1).

Não houve correlação significativa entre o Kt/V e o consumo de energia $(r=0,033 ; p=0,851)$, proteína ( $r=-$ $0,212 ; p=0,229)$ e fósforo $(r=-0,326 ; p=0,060)$. Contudo, houve correlação negativa estatisticamente significativa entre o valor da adequação de diálise e a ingestão de potássio $(r=-0,453 ; p=0,007)$.

Os alimentos que mais contribuíram para o consumo de fósforo foram a carne bovina, o leite, o feijão, o pão e o frango (tabela 2). Em relação ao consumo de potássio, os alimentos que mais contribuíram para a sua ingestão foram o café, o feijão, a carne bovina, o leite e o pão (tabela 3).

No presente estudo, 5,9\% dos pacientes apresentaram níveis séricos de fósforo abaixo do recomendado; $44,1 \%$ apresentaram níveis adequados e $50,0 \%$ acima do recomendado. Para o potássio, 41,2\% dos mesmos apresentaram níveis adequados e 58,8\% níveis elevados, não havendo correlação estatisticamente significativa entre o consumo de fósforo e potássio e seus níveis séricos $(r=0,030 ; p=0,867$ e $r=-$ 0,$116 ; p=0,513$, respectivamente).

\section{DISCUSSÃO}

A maioria dos pacientes em estudo apresentou peso adequado, embora o percentual de baixo peso seja considerável. Biavo et al. ${ }^{16}$, ao descreverem os aspectos nutricionais e epidemiológicos de pacientes com DRC em tratamento hemodialítico no Brasil em 2010, estabeleceram uma prevalência de baixo peso de 8,1\% para os pacientes com idade entre 18 e 59 anos e de $32,9 \%$ para aqueles com idade igual ou superior a 60 anos, utilizando o IMC como método de avaliação do estado nutricional, valores que se assemelham ao presente estudo. Embora o IMC não deva ser utilizado isoladamente como único parâmetro para a avaliação do estado nutricional, tal parâmetro constitui-se em um método apropriado para predizer a desnutrição nesses pacientes $^{17}$.

Em relação ao consumo alimentar, Pinto et al. ${ }^{18}$, ao identificarem a associação entre a ingestão de energia, proteína e fósforo em pacientes renais crônicos em tratamento dialítico, apontaram um consumo energético de $28,0 \mathrm{kcal} / \mathrm{kg}, 1,1 \mathrm{~g} / \mathrm{kg}$ de proteína e 958,0 mg de fósforo, valores superiores ao presente estudo. Ribeiro et al. ${ }^{19}$ demonstraram que a ingestão média de potássio. 
TABELA 1 - Caracterização e distribuição do consumo de energia, proteína, fósforo e potássio de uma amostra de pacientes com doença renal crônica em hemodiálise.

\begin{tabular}{|c|c|c|c|c|c|c|}
\hline Variável & $\mathbf{n}$ & $\%$ & Energia (kcal/kg) & Proteína $(\mathbf{g} / \mathbf{k g})^{\star}$ & Fósforo $(\mathrm{mg})^{\star}$ & Potássio $(\mathrm{mg})^{\star}$ \\
\hline \multicolumn{7}{|l|}{ Idade } \\
\hline$<60$ anos & 18 & 52,9 & $20,8 \pm 7,0$ & $0,9 \pm 0,4$ & $617,1 \pm 244,1$ & $1400,6 \pm 477,1$ \\
\hline$\geq 60$ anos & 16 & 47,1 & $17,0 \pm 4,5$ & $0,8 \pm 0,2$ & $607,2 \pm 178,5$ & $1399,8 \pm 445,2$ \\
\hline \multicolumn{7}{|l|}{ Sexo } \\
\hline Masculino & 22 & 64,7 & $20,0 \pm 5,5$ & $1,0 \pm 0,4$ & $697,9 \pm 181,7$ & $1563,6 \pm 453,1$ \\
\hline Feminino & 12 & 35,3 & $17,1 \pm 7,2$ & $0,7 \pm 0,2$ & $455,8 \pm 176,5$ & $1100,7 \pm 280,8$ \\
\hline \multicolumn{7}{|l|}{ Escolaridade } \\
\hline$\leq 8$ anos & 19 & 55,9 & $17,6 \pm 4,8$ & $0,9 \pm 0,3$ & $601,6 \pm 204,1$ & $1403,5 \pm 427,5$ \\
\hline$>8$ anos & 15 & 44,1 & $20,8 \pm 7,4$ & $0,9 \pm 0,4$ & $626,2 \pm 229,4$ & $1396,0 \pm 503,6$ \\
\hline \multicolumn{7}{|l|}{ Estado civil } \\
\hline Com cônjuge & 24 & 70,6 & $18,9 \pm 5,4$ & $0,9 \pm 0,4$ & $648,8 \pm 221,6$ & $1493,4 \pm 464,3$ \\
\hline Sem cônjuge & 10 & 29,4 & $19,3 \pm 8,1$ & $0,8 \pm 0,3$ & $525,3 \pm 168,1$ & $1176,4 \pm 361,2$ \\
\hline \multicolumn{7}{|l|}{ Raça } \\
\hline Branca & 22 & 64,7 & $18,9 \pm 6,2$ & $0,8 \pm 0,3$ & $600,2 \pm 209,9$ & $1369,9 \pm 425,7$ \\
\hline Não-branca & 12 & 35,3 & $19,1 \pm 6,4$ & $1,0 \pm 0,4$ & $634,9 \pm 224,9$ & $1455,7 \pm 520,3$ \\
\hline \multicolumn{7}{|l|}{ Renda } \\
\hline 1 a $3 \mathrm{SM}$ & 19 & 55,9 & $18,6 \pm 5,0$ & $0,9 \pm 0,3$ & $619,5 \pm 176,7$ & $1450,7 \pm 440,4$ \\
\hline 3 a $5 \mathrm{SM}$ & 8 & 23,5 & $17,1 \pm 6,2$ & $0,9 \pm 0,4$ & $561,8 \pm 234,6$ & $1207,5 \pm 398,4$ \\
\hline $5 \mathrm{SM}$ ou mais & 7 & 20,6 & $22,2 \pm 8,6$ & $0,9 \pm 0,5$ & $651,1 \pm 292,0$ & $1483,3 \pm 554,5$ \\
\hline \multicolumn{7}{|l|}{ Estado nutricional } \\
\hline Baixo peso & 6 & 17,6 & $15,6 \pm 3,2$ & $0,7 \pm 0,2$ & $500,6 \pm 192,4$ & $1237,5 \pm 456,7$ \\
\hline Peso adequado & 17 & 50,0 & $20,2 \pm 6,5$ & $0,9 \pm 0,3$ & $632,5 \pm 190,3$ & $1410,6 \pm 427,5$ \\
\hline Excesso de peso & 11 & 32,4 & $19,1 \pm 6,7$ & $0,9 \pm 0,4$ & $642,5 \pm 251,9$ & $1472,9 \pm 515,4$ \\
\hline \multicolumn{7}{|c|}{ Período de tratamento } \\
\hline Matutino & 7 & 20,6 & $22,3 \pm 7,3$ & $1,1 \pm 0,4$ & $716,6 \pm 257,1$ & $1583,2 \pm 650,3$ \\
\hline Vespertino & 13 & 38,2 & $17,5 \pm 6,8$ & $0,7 \pm 0,2$ & $548,6 \pm 166,0$ & $1256,2 \pm 350,6$ \\
\hline Noturno & 14 & 41,2 & $18,7 \pm 4,7$ & $0,9 \pm 0,3$ & $619,7 \pm 220,9$ & $1442,4 \pm 420,7$ \\
\hline \multicolumn{7}{|l|}{ Tempo de tratamento } \\
\hline 6 a 18 meses & 17 & 50,0 & $20,7 \pm 5,4$ & $1,0 \pm 0,4$ & $679,5 \pm 224,4$ & $1503,2 \pm 498,6$ \\
\hline 18 a 30 meses & 7 & 20,6 & $17,3 \pm 6,3$ & $0,8 \pm 0,3$ & $574,9 \pm 226,8$ & $1353,2 \pm 537,2$ \\
\hline 30 meses ou mais & 10 & 29,4 & $17,3 \pm 7,1$ & $0,7 \pm 0,2$ & $524,7 \pm 154,0$ & $1258,1 \pm 285,7$ \\
\hline \multicolumn{7}{|l|}{ Pagamento } \\
\hline SUS & 29 & 85,3 & $18,8 \pm 6,5$ & $0,8 \pm 0,4$ & $592,9 \pm 216,6$ & $1367,0 \pm 454,4$ \\
\hline Convênio & 5 & 14,7 & $19,9 \pm 4,1$ & $1,0 \pm 0,3$ & $725,6 \pm 159,9$ & $1592,5 \pm 314,6$ \\
\hline
\end{tabular}

Sem cônjuge= separado(a)/divorciado(a), viúvo(a) ou solteiro(a) sem relacionamento atual.

SM= salário-mínimo; SUS= Sistema Único de Saúde.

*Diferença estatisticamente significativa no consumo alimentar de acordo com a variável sexo.

de sua amostra em estudo correspondeu a 1981,8 $\mathrm{mg} / \mathrm{dia}$, igualmente superior a presente pesquisa. $\mathrm{O}$ consumo reduzido nesses pacientes pode estar relacionado à falta de apetite e fatores hormonais e inflamatórios, devido à alterações nos níveis séricos de grelina, leptina, obestatina e acyl-grelina ${ }^{20,21}$. No presente estudo não houve associação entre a inadequação da diálise e o baixo consumo de nutrientes, sendo importante ressaltar a importância do acompanhamento nutricional, uma vez que intervenções realizadas para a promoção da ingestão de energia reduzem o risco de mortalidade por desnutrição ${ }^{22}$

Antunes et al. $^{23}$, ao avaliarem a influência dos parâmetros nutricionais sobre a sobrevida de pacientes em diálise demonstraram que o consumo inferior a 1,2 g/kg/dia de proteínas constitui-se como um preditor 
TABELA 2 - Contribuição para o consumo total de fósforo dos alimentos consumidos por uma amostra de pacientes com doença renal crônica em hemodiálise.

\begin{tabular}{ccccc}
\hline Classificação & Alimento/preparação & $\mathbf{n}$ & $\%$ & \% acumulada \\
\hline 1 & Carne bovina & 29 & 18,1 & 18,1 \\
2 & Leite & 29 & 15,2 & 33,3 \\
3 & Feijão & 32 & 12,8 & 46,1 \\
4 & Pão & 34 & 11,4 & 57,5 \\
5 & Frango & 19 & 10,0 & 72,5 \\
6 & Peixe & 7 & 5,0 & 77,2 \\
7 & Arroz & 33 & 80,6 \\
8 & Macarrão & 20 & 3,7 & 83,8 \\
9 & Queijos & 14 & 3,2 & 86,0 \\
10 & Café & 33 & 2,2 & 87,5 \\
11 & Ovo & 11 & 1,5 & 89,0 \\
12 & Bolos & 11 & 1,5 & 90,1 \\
13 & Embutidos & 10 & 1,1 & 91,2 \\
14 & logurtes & 2 & 1,1 & 92,1 \\
15 & Carne suína & 3 & 0,9 & 92,8 \\
16 & Banana & 14 & 0,7 & 93,5 \\
17 & Sopas & 4 & 0,7 & 94,2 \\
18 & Biscoito doce & 4 & 0,7 & 94,8 \\
19 & Batata & 6 & 0,6 & 95,3
\end{tabular}

Classificação= alimento que mais contribuiu para o consumo total de fósforo para o que menos contribuiu em relação ao total consumido pela amostra em estudo. $n=$ número de pacientes que relataram o consumo do alimento. \%= percentual de contribuição do alimento em relação ao total de fósforo consumido pela amostra em estudo.

independente de mortalidade. Por sua vez, Naylor et al. $^{24}$, ao realizarem uma revisão de literatura com o objetivo de elaborar diretrizes sobre a necessidade proteica de pacientes em hemodiálise chegaram à conclusão de que a ingestão de $1,1 \mathrm{~g} / \mathrm{kg} / \mathrm{dia}$ desse macronutriente é capaz de atender as necessidades dos mesmos e evitar a desnutrição proteica. No entanto, no presente estudo o consumo de proteínas permaneceu inferior a esse valor, correspondendo a $0,9 \mathrm{~g} / \mathrm{kg} / \mathrm{dia}$, ressaltando a necessidade de intervenção nutricional, uma vez que essa ingestão insuficiente está relacionada a uma maior mortalidade.

Em sua grande maioria, os alimentos ricos em fósforo são igualmente fonte de proteínas. Uma vez que a DEP está relacionada a maior mortalidade, a redução do consumo de alimentos proteicos, visando a diminuição do consumo de fósforo, não deve ser estimulada. Para tanto, métodos que podem ser utilizados incluem a oferta de suplementos nutricionais com alto teor de energia e proteínas, mas com teores reduzidos do mesmo e o uso correto de quelantes desse mineral ${ }^{25}$.
Gutiérrez e Wolf $^{26}$ sugerem que deve ser estimulada a redução da ingestão de alimentos aditivados de fósforo e aqueles com elevada relação fósforo/proteína, em favor do consumo de alimentos proteicos com baixa biodisponibilidade desse mineral. Contudo, os aditivos de fósforo, amplamente utilizados em produtos industrializados, podem possuir biodisponibilidade de até $100 \%$, devendo ser orientado a esses pacientes que evitem o consumo dos mesmos ${ }^{27}$. Deve-se destacar, nesse caso, a limitação dos programas de avaliação do consumo alimentar existentes, uma vez que os mesmos não consideram 0 fósforo contido nos aditivos alimentares, com 0 agravante de que a biodisponibilidade dos mesmos é maior.

Considerando-se a importância da ingestão de proteínas e a necessidade do controle dos níveis séricos de fósforo, programas de educação nutricional que visem a promoção do consumo do primeiro e que priorizem alimentos com baixos teores do segundo devem ser realizados. Nisio et al. $^{28}$, ao avaliarem o impacto de um programa de educação nutricional sobre 
TABELA 3 - Contribuição para o consumo total de potássio dos alimentos consumidos por uma amostra de pacientes com doença renal crônica em hemodiálise.

\begin{tabular}{|c|c|c|c|c|}
\hline Classificação & Alimento/preparação & $\mathbf{n}$ & $\%$ & $\%$ acumulada \\
\hline 1 & Café & 33 & 16,8 & 16,8 \\
\hline 2 & Feijão & 32 & 16,4 & 33,2 \\
\hline 3 & Carne bovina & 29 & 12,7 & 45,9 \\
\hline 4 & Leite & 29 & 10,9 & 56,8 \\
\hline 5 & Pão & 34 & 5,3 & 62,1 \\
\hline 6 & Frango & 19 & 5,2 & 67,3 \\
\hline 7 & Macarrão & 20 & 3,9 & 71,2 \\
\hline 8 & Peixe & 7 & 3,4 & 74,6 \\
\hline 9 & Banana & 14 & 3,4 & 78,0 \\
\hline 10 & Batata & 10 & 1,7 & 79,7 \\
\hline 11 & Arroz & 33 & 1,7 & 81,4 \\
\hline 12 & Sopas & 4 & 1,4 & 82,8 \\
\hline 13 & Alface & 20 & 1,3 & 84,1 \\
\hline 14 & Manga & 8 & 1,2 & 85,3 \\
\hline 15 & Laranja & 7 & 1,1 & 86,4 \\
\hline 16 & Mamão & 3 & 1,1 & 87,5 \\
\hline 17 & Tomate & 9 & 0,9 & 88,4 \\
\hline 18 & Bolos & 11 & 0,8 & 89,2 \\
\hline 19 & Embutidos & 10 & 0,8 & 90,0 \\
\hline 20 & logurtes & 2 & 0,8 & 90,8 \\
\hline 21 & Maxixe & 2 & 0,7 & 91,5 \\
\hline 22 & Quiabo & 3 & 0,7 & 92,2 \\
\hline 23 & Maçã & 8 & 0,6 & 92,8 \\
\hline 24 & Escarola & 5 & 0,6 & 93,4 \\
\hline 25 & Carne suína & 3 & 0,6 & 94,0 \\
\hline 26 & Cenoura & 7 & 0,6 & 94,6 \\
\hline 27 & Mandioca & 2 & 0,6 & 95,2 \\
\hline
\end{tabular}

Classificação= alimento que mais contribuiu para o consumo total de potássio para o que menos contribuiu em relação ao total consumido pela amostra em estudo. $n=$ número de pacientes que relataram o consumo do alimento. \%= percentual de contribuição do alimento em relação ao total de potássio consumido pela amostra em estudo.

o conhecimento a respeito de fósforo e a fosfatemia de pacientes em diálise, demonstraram que a aplicação do mesmo resultou em melhora do conhecimento e redução de suas concentrações séricas, particularmente nos pacientes hiperfosfatêmicos.

Embora as frutas, verduras e legumes serem reconhecidamente as principais fontes desse mineral, o presente estudo identificou que esses alimentos não contribuíram de forma significativa em relação ao consumo total desse mineral, visto que a ingestão dos mesmos apresentou-se baixa na amostra em estudo. Até o presente momento não foram identificados estudos que realizaram intervenções nutricionais visando a redução dos níveis séricos de potássio. 0 remolho e determinados métodos de cocção mostraram- se eficazes na diminuição do teor desse mineral em hortaliças ${ }^{29}$, podendo ser utilizados como estratégias para a redução do consumo do mesmo.

A não-correlação entre a ingestão e os níveis séricos de fósforo e potássio foi esperada, considerando-se que fatores extrínsecos à dieta influenciam o aumento dos níveis séricos desses minerais, como a baixa adesão ao uso de quelantes de fósforo e a eficiência da diálise, que estão relacionadas com a hiperfosfatemia ${ }^{30}$, e a acidose metabólica, lise celular, deficiência de insulina, uso de betabloqueadores e diuréticos poupadores de potássio e constipação, que se associam com a hipercalemia ${ }^{31}$. Também deve ser considerado que o consumo alimentar dos pacientes possa ter sido alterado ou subrelatado, uma vez que os pacientes sabiam que 
estavam sendo avaliados ${ }^{32}$.

Não foram identificados estudos que avaliaram o consumo alimentar de pacientes em hemodiálise de acordo com as variáveis utilizadas na presente pesquisa, ressaltando a necessidade desse tipo de estudo. Um dos fatores limitantes do presente estudo foi a baixa adesão dos pacientes, fazendo com que o número de participantes fosse relativamente baixo, indicando a necessidade de mais estudos que avaliem a ingestão alimentar dos mesmos com a utilização de métodos que não dependam da adesão do paciente após o primeiro contato.

Os resultados do presente estudo demonstraram que portadores de DRC em tratamento hemodialítico apresentaram consumo abaixo do recomendado para energia, proteína, fósforo e potássio. Foi observada diferença estatisticamente significativa na ingestão de proteína, fósforo e potássio pela variável sexo, sendo que os pacientes do sexo masculino apresentaram maior consumo dos mesmos. Não houve associação entre a inadequação da diálise e o baixo consumo de nutrientes, bem como não houve correlação entre a ingestão de fósforo e potássio e seus respectivos níveis séricos.

Assim, medidas de educação nutricional devem ser continuamente realizadas, para que a ingestão dos mesmos se adeque às recomendações $e$ as comorbidades decorrentes do consumo inadequado sejam evitadas.

\section{REFERÊNCIAS}

1. Kirsztajn GM, Bastos MG, Andriolo A. Dia Mundial do Rim 2011 - Proteinúria e creatinina sérica: testes essenciais para diagnóstico de doença renal crônica. J Bras Patol Med Lab. 2011; 47(2):100-3.

2. Sesso RC, Lopes AA, Thomé FS, Lugon JR, Watanabe $Y$, Santos DR. Relatório do Censo Brasileiro de Diálise Crônica 2012. J Bras Nefrol. 2014; 36(1):48-53.

3. National Kidney Foundation. Kidney Disease Outcomes Quality Initiative. Clinical practice guidelines for chronic kidney disease: evaluation, classification and stratification. Am J Kidney Dis. 2002;39(1):S1-266

4. Bastos MG, Bregman R, Kirsztajn GM. Doença renal crônica: frequente e grave, mas também prevenível e tratável. Rev Assoc Med Bras. 2010; 56(2):248-53.

5. Nerbass FB, Cuppari L. Hemodiálise. In: Cuppari L, Avesani CM, Kamimura MA. Nutrição na doença renal crônica. Barueri: Manole; 2013. p.247-69.

6. National Kidney Foundation. Kidney Disease Outcomes Quality Initiative. Clinical practice guidelines for nutrition in chronic renal failure. Am J Kidney Dis. 2000; 35(2):17-103.
7. Carvalho AB, Cuppari L. Controle da hiperfosfatemia na DRC. J Bras Nefrol. 2011; 33(1):191-6.

8. Kamimura MA, Avesani CM, Draibe SA, Cuppari L. Gasto energético de repouso em pacientes com doença renal crônica. Rev Nutr. 2008; 21(1):75-84.

9. Huml AM, Sullivan CM, Leon JB, Sehgal AR. The adequacy of phosphorus binder prescriptions among American hemodialysis patients. Ren Fail. 2012; 34(10):1258-63.

10. Rachoin JS, Weisberg LS. How should dialysis fluid be individualized for the chronic hemodialysis patient? Potassium. Semin Dial. 2008; 21(3):223-5.

11. Fouque D, Vennegoor M, Wee PT, Wanner C, Basci A, Canaud B, Haage P, Konner K, Kooman J, Martin-Malo A, Pedrini L, Pizzarelli F, Tattersall J, Tordoir J, Vanholder R. EBPG guideline on nutrition. Nephrol Dial Transplant. 2007; 22(2):ii45-87.

12. World Health Organization. Obesity: Preventing and Managing the Global Epidemic. Geneva: WHO; 2000

13. Organização Pan-Americana da Saúde. XXXVI Reunión del Comitê Asesor de Investigaciones en Salud Encuestra Multicêntrica - Salud Beinestar y Envejecimiento (SABE) en América Latina e el Caribe Informe Preliminar [acesso em 17 mar 2014]. 2002. Disponível em: http://www.opas.org/program/sabe.htm

14. Pinheiro ABV, Lacerda EMA, Benzecry EH, Gomes MCS, Costa VM. Tabela para Avaliação de Consumo Alimentar em Medidas Caseiras. 5. ed. São Paulo: Atheneu; 2008.

15. Block G, Dresser CM, Hartman AM, Carroll MD. Nutrient sources in the American diet: quantitative data from the NHANES II survey. I. Vitamins and minerals. Am J Epidemiol. 1985; 122(1):13-26.

16. Biavo BMM, Tzanno-Martins C, Cunha LM, Araujo ML, Ribeiro MMC, Sachs A, Uezima CBB, Draibe SA, Rodrigues CIS, Barros EJG. Aspectos nutricionais e epidemiológicos de pacientes com doença renal crônica submetidos a tratamento hemodialítico no Brasil, 2010. J Bras Nefrol. 2012; 34(3):206-15.

17. Chen J, Peng $\mathrm{H}$, Yuan Z, Zhang K, Xiao L, Huang J, Wang J, Huang $\mathrm{H}$. Combination with anthropometric measurements and MQSGA to assess nutritional status in Chinese hemodialysis population. Int J Med Sci. 2013; 10(8):974-80

18. Pinto DE, Ullmann LS, Burmeister MM, Antonello ICF, Pizzato A. Associações entre ingestão energética, proteica e de fósforo em pacientes portadores de doença renal crônica em tratamento hemodialítico. J Bras Nefrol. 2009; 31(4):269-74.

19. Ribeiro MMC, Araújo ML, Netto MP, Cunha LM. Impacto do hábito de jantar sobre o perfil dietético de pacientes em hemodiálise. J Bras Nefrol. 2011;33(1):69-77.

20. Mafra D, Jolivot A, Chauveau P, Drai J, Azar R, Michel C, Fouque D. Are ghrelin and leptin involved in food intake and body mass index in maintenance hemodialysis? J Ren Nutr. 2010; 20(3):151-7.

21. Oner-lyidogan $Y$, Gurdol $F$, Kocak $H$, Oner $P$, CetinalpDemircan P, Caliskan Y, Kocak T, Turkmen A. Appetiteregulating hormones in chronic kidney disease patients. $J$ Ren Nutr. 2011; 21(4):316-21.

22. Molfino A, Chiappini MG, Laviano A, Ammann T, Bollea MR, Alegiani F, Rossi-Fanelli F, Muscaritoli M. Effect of intensive nutritional counseling and support on clinical outcomes of hemodialysis patients. Nutrition. 2012; 28(10):1012-5.

23. Antunes AA, Vannini FD, Silveira LVA, Martin LC, Barretti $P$, Caramori JC. Influence of protein intake and muscle mass on survival in chronic dialysis patients. Ren Fail. 2010; 32(9):1055-9.

24. Naylor HL, Jackson $H$, Walker $G H$, Macafee $S$, Magee K, 
Hooper L, Stewart L, MacLaughlin HL, Renal Nutrition Group of the British Dietetic Association, British Dietetic Association. British Dietetic Association evidence-based guidelines for the protein requirements of adults undergoing maintenance haemodialysis or peritoneal dialysis. J Hum Nutr Diet. 2013; 26(4):315-28.

25. González-Parra E, Gracia-Iguacel C, Egido J, Ortiz A. Phosphorus and nutrition in chronic kidney disease. Int $J$ Nephrol. 2012; 2012:597605.

26. Gutiérrez OM, Wolf M. Dietary phosphorus restriction in advanced chronic kidney disease: merits, challenges, and emerging strategies. Semin Dial. 2010; 23(4):401-6.

27. Benini O, D'Alessandro C, Gianfaldoni D, Cupisti A. Extraphosphate load from food additives in commonly eaten foods: a real and insidious danger for renal patients. J Ren Nutr. 2011; 21(4):303-8.

28. Nisio JM, Bazanelli AP, Kamimura MA, Lopes MGG, Ribeiro FSM, Vasselai P, Oliveira CMV, Manfredi SR, Canziani ME, Draibe SA, Cuppari L. Impacto de um programa de educação nutricional no controle da hiperfosfatemia de pacientes em hemodiálise. J Bras Nefrol. 2007; 29(3):152-7.
29. Copetti C, Oliveira VR, Kirinus P. Avaliação da redução de potássio em hortaliças submetidas a diferentes métodos de cocção para possível utilização na dietoterapia renal. Rev Nutr. 2010;23(5):831-8.

30. Karamanidou C, Clatworthy J, Weinman J, Horne R. A systematic review of the prevalence and determinants of nonadherence to phosphate binding medication in patients with end-stage renal disease. BMC Nephrol. 2008;9(2).

31. Rocha PN. Hipercalemia. J Bras Nefrol. 2009;31(1):5.

32. Fisberg RM, Marchioni DML, Colucci ACA. Avaliação do consumo alimentar e da ingestão de nutrientes na prática clínica. Arq Bras Endocrinol Metab. 2009; 53(5):617-24.

\section{Endereço para correspondência:}

Alisson Diego Machado

Rua Marisa $\mathrm{n}^{\circ} 121$

Barueri/SP - CEP 06436-190

Telefone: +55 1141945355/+55 11980541963

Email: alissondiegomachado@hotmail.com 